\title{
SYMMETRY AND ELECTRONIC STRUCTURE OF NOBLE METAL NANOPARTICLES AND THE ROLE OF RELATIVITY
}

\author{
Hannu Häkkinen \\ Department of Physics, NanoScience Center, \\ FIN-40014 University of Jyväskylä, Finland \\ Michael Moseler \\ Fraunhofer Institute for mechanics of materials, \\ Wohlerstraße 11, D-79108 Freiburg, Germany \\ Oleg Kostko, Nina Morgner, Margarita Astruc Hoffmann, and Bernd v. Issendorfl* \\ Fakultät für Physik, Universität Freiburg, \\ Stefan-Meier-Straße 21, D-79104 Freiburg, Germany
}

(Dated: July 30, 2021)

\begin{abstract}
High resolution photoelectron spectra of cold mass selected $\mathrm{Cu}_{n}^{-}, \mathrm{Ag}_{n}^{-}$and $\mathrm{Au}_{n}^{-}$with $\mathrm{n}=53-58$ have been measured at a photon energy of $6.42 \mathrm{eV}$. The observed electron density of states is not the expected simple electron shell structure, but seems to be strongly influenced by electron-lattice interactions. Only $\mathrm{Cu}_{55}^{-}$and $\mathrm{Ag}_{55}^{-}$exhibit highly degenerate states. This is a direct consequence of their icosahedral symmetry, as is confirmed by density functional theory calculations. Neighboring sizes exhibit perturbed electronic structures, as they are formed by removal or addition of atoms to the icosahedron and therefore have lower symmetries. Gold clusters in the same size range show completely different spectra with almost no degeneracy, which indicates that they have structures of much lower symmetry. This behaviour is related to strong relativistic bonding effects in gold, as demonstrated by ab initio calculations for $\mathrm{Au}_{55}^{-}$.
\end{abstract}

PACS numbers: $33.60 . \mathrm{Cv}, 36.40 . \mathrm{Cg}, 73.22 .-\mathrm{f}$

*Electronic address: bernd.von.issendorff@uni-freiburg.de 
Understanding the energetically most favorable structures that aggregates of metal atoms inherently adopt during their formation process is one of the long-standing issues in the science of clusters and nanoparticles 1, 2]. The atomic structure and its symmetry are intimately related to the electronic structure, which in turn defines the electrical, optical and chemical properties of the particle. Resolving the atomic structures of nanoclusters therefore represents an important preliminary for their controlled use in future nanotechnologies. Noble metal clusters and nanoparticles have attracted much attention recently, as they are considered promising candidates for applications e.g. in catalysis [3, 4], labeling [5] or photonics [6]. Two size ranges of these particles have been characterized in detail: small clusters with up to 21 atoms have been studied by high resolution photoelectron and optical spectroscopy as well as a number of theoretical studies 7, 8, 19, 10, 11, 12, 13, 14]. The results show that in this still rather "molecular" size range the clusters tend to adopt a variety of low symmetry structures (the tetrahedral $\mathrm{Au}_{20}^{-}$being a prominent exception [14]). Much larger particles, with hundreds or thousands of atoms, have been studied by electron microscopy and electron diffraction [15, 16]. It turned out that these particles adopt wellordered structures with surprisingly diverse overall symmetries. Icosahedral, decahedral and (bulk-like) octahedral forms are often observed at the same time, with the preferred structural motif depending sensitively on the particle formation process. Experimental structural information for medium sized (around 50 atoms and up) $\mathrm{Cu}, \mathrm{Ag}$ and $\mathrm{Au}$ clusters was scarce up to now. Measurements of ionization potentials [17] and UV-photoelectron spectroscopy studies 18] gave evidence for the existence of some free electron shell structure, which lead to the general perception that the properties of noble metal clusters in this size range can be fully explained by the free electron model.

We have performed high resolution UV-photoelectron spectroscopy on free, cold, size selected noble metal clusters. The setup is the same as used in earlier experiments [20]. Copper, silver and gold clusters were produced by magnetron discharge sputtering of a metal target inside a liquid nitrogen cooled aggregation tube, through which a mixture of helium and argon is flowing at a pressure of about 0.5 mbar. As the discharge produces many charged condensation seeds, a large portion of the clusters formed in the cold gas flow is negatively (or positively) charged. After having covered a distance of $25 \mathrm{~cm}$ the gas with the clusters inside expands into the vacuum through an adjustable aperture (typically $4 \mathrm{~mm}$ diameter). The clusters pass a skimmer and are inserted into a double-reflectron time-of- 


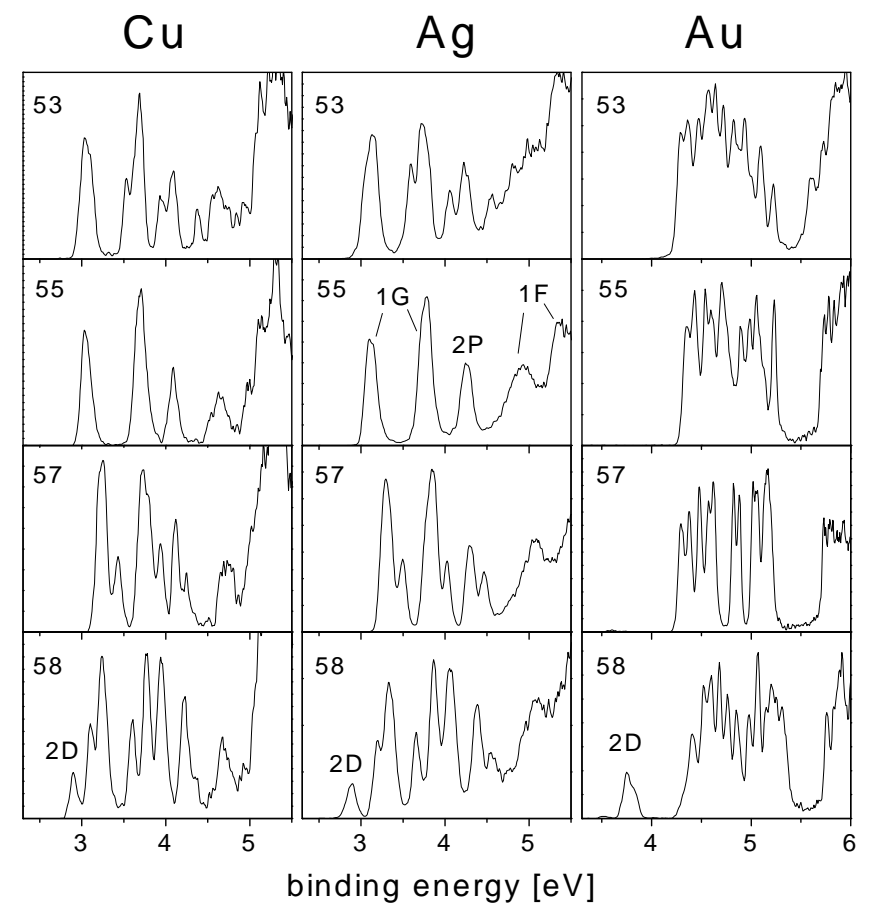

FIG. 1: Photoelectron spectra of $\mathrm{Cu}_{N}^{-}, \mathrm{Ag}_{N}^{-}$and $\mathrm{Au}_{N}^{-}(\mathrm{N}=53,55,57,58)$ obtained at a photon energy of $6.42 \mathrm{eV}$.

flight mass spectrometer, which is used to select a single cluster size. These clusters are decelerated and inserted into a magnetic bottle time-of-flight photoelectron spectrometer, where they are irradiated by photons from an ArF-excimer laser (hv=6.42 eV). The flight time distribution of the emitted electrons is measured and converted into a binding energy distribution. The electron spectrometer has an energy resolution of about $\mathrm{E} / \mathrm{dE}=40$. It has been calibrated by measuring the known spectra of the monomer anions, which leads to an error of the energy axis of less than $30 \mathrm{meV}$. Typically, the photoelectron spectra have been averaged over 30000 laser shots at a repetition rate of $100 \mathrm{~Hz}$. We estimate the temperature of the clusters to be $200 \pm 50 \mathrm{~K}$.

The spectra obtained for $\mathrm{Cu}_{n}^{-}, \mathrm{Ag}_{n}^{-}$and $\mathrm{Au}_{n}^{-}$with $\mathrm{n}=53-58$ are shown in Fig. 1. In principle these are direct images of the electronic density of states. In the bulk the electronic structure of nobel metals is characterized by a half-filled and rather free-electron like band formed from the atomic s-orbitals, intersected some eV below the Fermi energy by the so-called d-band, which is formed from the rather localized atomic d-orbitals. As has been discussed in detail already by Taylor et al. 18] this structure is clearly visible in the 
photoelectron spectra: the uppermost part of the distributions can be identified as being dominantly s-electron derived, while the onset of the d-bands can be observed roughly 2-3 $\mathrm{eV}$ below the uppermost state. The s-band part of the electronic density of states should therefore exhibit the same discrete structure as observed e.g. for alkali clusters [1, 19]. The 57-atom cluster anions contain 58 valence s-electrons, and in the spherical droplet approximation should have the closed shell configuration $1 \mathrm{~S}^{2} 1 \mathrm{P}^{6} 1 \mathrm{D}^{10} 2 \mathrm{~S}^{2} 1 \mathrm{~F}^{14} 2 \mathrm{P}^{6} 1 \mathrm{G}^{18}$ (for clarity we refer to free electron angular momentum states with a capital letter). Adding one atom should lead to the appearance of a singly occupied 2D orbital. Indeed for all three metals a new peak appears for size 58, which indicates the formation of a new shell. However, the free electron model also predicts that in the section of the s-band visible here only four shells should be present: 1F, 2P, 1G and 2D. The structure of the photoelectron spectra obviously is more complicated than that, which hints at a relatively strong perturbation of the shell structure by electron-lattice interaction. The only clusters exhibiting a clear structure of highly degenerate states are $\mathrm{Cu}_{55}^{-}$and $\mathrm{Ag}_{55}^{-}$. Such degeneracies can only be produced by a highly symmetric atomic structure. $\mathrm{Au}_{55}^{-}$, on the other hand, exhibits a complex spectrum without significant level bunching; it therefore seems not to adopt any of the atomic symmetries that lead to pronounced degeneracy of electron shells. This is in accordance with earlier mass spectroscopic measurements which demonstrated that 55 is a strong atomic magic number for silver, but not for gold [21, 22].

We will now discuss these findings in the light of our theoretical results. The atomic and electronic structures of silver and gold clusters were calculated by density functional theory in combination with Born-Oppenheimer molecular dynamics 23], including self-consistent gradient corrections [24]. The interaction of the $\mathrm{d}^{10} \mathrm{~s}^{1}$ valence electrons of $\mathrm{Ag}$ and $\mathrm{Au}$ with the atomic cores was described by scalar-relativistic norm-conserving pseudopotentials [25]. For $\mathrm{Au}$ a non-relativistic pseudopotential was used as well, in order to check the influence of relativity. The Kohn-Sham orbitals were expanded in a plane wave basis with a kinetic energy cutoff of 62 Ry. The method does not employ a supercell, i.e., a periodic image of the atoms, and is thus suitable for accurate calculations for charged finite clusters[23]. As a full simulated-annealing scheme with ab-initio determination of the electronic structure at each dynamic step still is computationally too expensive for this size of d-metal clusters (having a total number of valence electrons of about 600), we adopted an alternative strategy. We made use of the already existing knowledge about possible structures of nanosized metal 
clusters, which has been obtained by simulated annealing and genetic algorithm optimization of clusters described by classical pairwise and many-atom interatomic potentials. An extensive collection of such structures, known as the Cambridge Cluster Database (CCD), is publicly available on the World Wide Web[26]. For the structural optimization of $\mathrm{Ag}_{55}^{-}$ and $\mathrm{Au}_{55}^{-}$we chose 6 candidates representing different structural motifs: closed atomic shell icosahedral (ICO), decahedral (DECA), and cuboctahedral (CUBO) structures, and the optimum structures found for classical Sutton-Chen (SC), glue, and Morse potentials. These geometries were then optimized by ab initio molecular dynamics simulations, allowing the clusters to relax for 1 ps under the influence of a global friction force. In none of the cases this relaxation lead to a severe change of the overall structure.

The optimized structures and their electronic densities of states (DOS) as well as the measured spectra are shown in Fig. 2. One can see that only two of them, the icosahedron [27] and the cuboctahedron, show a clear shell structure like the one observed in the experiment. All other isomers exhibit much less distinct level bunching. Of the six structures considered, the icosahedral cluster ICO is the clear ground state. Its calculated DOS matches very well the experimental photoelectron spectrum. This allows us to identify the shells visible in the measured spectrum as the icosahedral orbitals $\mathrm{T}_{2 u}^{6} \mathrm{G}_{u}^{8} \mathrm{~T}_{1 u}^{6} \mathrm{H}_{g}^{10} \mathrm{G}_{g}^{6}$. These levels can be seen as being produced from the angular momentum eigenstates of the spherical droplet by crystal field splitting. The lattice of the cluster represents a perturbing potential of icosahedral symmetry. Group theory tells us 28] that such a perturbation does not lift the degeneracy of the $2 \mathrm{P}$ shell, but splits the $1 \mathrm{~F}$ and $1 \mathrm{G}$ shell into two subshells each, as indicated in Fig. 1. The separation of these subshells can therefore be taken as a measure for the strength of the electron lattice interaction. The cuboctahedral cluster, though exhibiting a DOS very similar to that of the ICO cluster, can safely be excluded to be present in the experiment because of its significantly higher energy. All other isomers will have a more complex DOS. So although we cannot exclude that other low symmetry isomers exist which are energetically closer to the ICO than the ones presented here, the purity of the experimental spectrum demonstrates that the icosahedron is by far the dominant structure for the $\mathrm{Cu}_{55}^{-}$and $\mathrm{Ag}_{55}^{-}$ clusters produced by our source.

As the atomic symmetry of these two clusters leads to the high degeneracy in their electronic structure 27], it is interesting to see how it will change if the symmetry is perturbed. In Fig. 1 one can observe that if the cluster size is increased or decreased by two atoms, the 


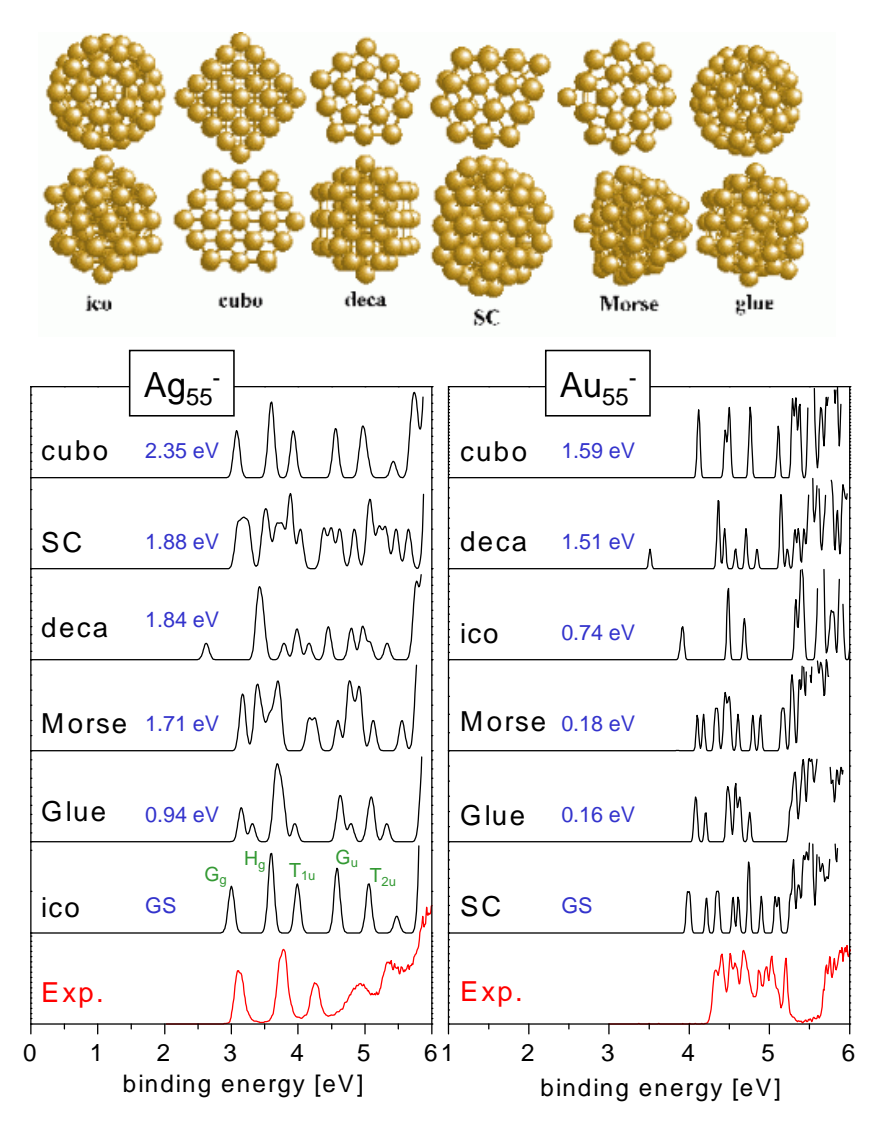

FIG. 2: Structures and density of states (DOS) of Ag and Au clusters obtained via density functional calculations. (a) Six candidate structures (displayed are the fully relaxed optimal structures for gold) for $\mathrm{Ag}_{55}^{-}$and $\mathrm{Au}_{55}^{-}$representing different structural motifs: closed atomic shell icosahedral (ICO), decahedral (DECA), and cuboctahedral (CUBO) structures, and clusters optimized previously by classical Sutton-Chen (SC), Glue, and short ranged Morse potentials (b) DOS of the six structures (black curves) compared to the experimental photoelectron spectra (red curves) for $\mathrm{Ag}$ (left panel) and Au (right panel). The numbers denote the energy difference to the most stable structure (GS).

three upper peaks in the spectra split up. This splitting can be most easily understood for the case of the $\mathrm{T}_{1 u}(2 \mathrm{P})$ orbital. Assuming that the geometries of the clusters are based on the 55-atom icosahedron, $\mathrm{Ag}_{53}^{-}$has two vacancies in the outermost atom shell and therefore a slightly oblate shape, whence $\mathrm{Ag}_{57}^{-}$has two adatoms and is prolate. In the case of a P-type orbital this will lead to a splitting into the $\mathrm{P}_{x}^{2} \mathrm{P}_{y}^{2} \mathrm{P}_{z}^{2}$ subshells, with 1:2 and 2:1 intensity ratios of the lower:higher binding energies for oblate and prolate shapes, respectively. This 
is exactly what one can observe in Fig. 1. The same can be seen in the theoretical spectra of $\mathrm{Ag}_{57}^{-}$in Fig. 3, where the calculated DOS of the three lowest energy isomers is shown. Interestingly the splitting of the shells is more pronounced in the calculation than in the experiment. This could be a temperature effect: in the calculation the geometries are fully relaxed at $0 \mathrm{~K}$; here the attached atoms produce some distortion of the icosahedral core and thereby some additional perturbation of the shell structure. In the experiment the finite cluster temperature (about 150-250 K) will probably lead to a larger average bond length of the surface atoms and therefore to a reduced effect on the icosahedral core. Additionally the attached atoms will probably be more mobile than the energy differences between the different isomers indicate. In order to check this we show a simple average of the three calculated spectra as well. The better agreement of this averaged spectrum with the measured one indicates that indeed more than one isomer is present in the experiment, which is probably due to a constant movement of the attached atoms on the icosahedral core.

Let us now turn to the case of gold. The experimental spectrum of the $\mathrm{Au}_{55}^{-}$cluster is drastically different from those of $\mathrm{Ag}_{55}^{-}$and $\mathrm{Cu}_{55}^{-}$(Fig. 1). The visible part of the "sband" is highly structured, but does not show any trace of a shell pattern. This can be taken as direct evidence that a free $\mathrm{Au}_{55}^{-}$does not adopt icosahedral or cuboctahedral symmetry. Indeed from the computations we find several low-symmetry structures (SC, GL, MO) for $\mathrm{Au}_{55}^{-}$below the ICO. The calculated DOS of these isomers are in a qualitative agreement with the measured spectra, exhibiting no significant level bunching. Perfect agreement cannot be expected, as spin-orbit coupling, which is not taken into account in the calculation, will further perturb the DOS. Additionally, one cannot assume that the SC isomer is the true ground state of $\mathrm{Au}_{55}^{-}$, given the vast amount of possible low-symmetry structures (in a previous calculation using semiempirical potentials 360 disordered isomers have been identified that were energetically favorable to the ICO [29]). Nevertheless both our experiment and our calculation give strong evidence for a low symmetry ground state of $\mathrm{Au}_{55}^{-}$. So why are gold clusters so different from silver or copper clusters? The answer is found by comparing the results of a nonrelativistic calculation with those of a scalarrelativistic one. It turns out that a fictitious, nonrelativistic $\mathrm{Au}_{55}^{-}$behaves very similar to $\mathrm{Cu}_{55}^{-}$or $\mathrm{Ag}_{55}^{-}$, having a clear ICO ground state and an almost identical DOS. Only in the scalar-relativistic calculation the lower symmetry isomers are preferred, which is due to a change in the nature of the interatomic bonding. The Au atom is known to be "the most 


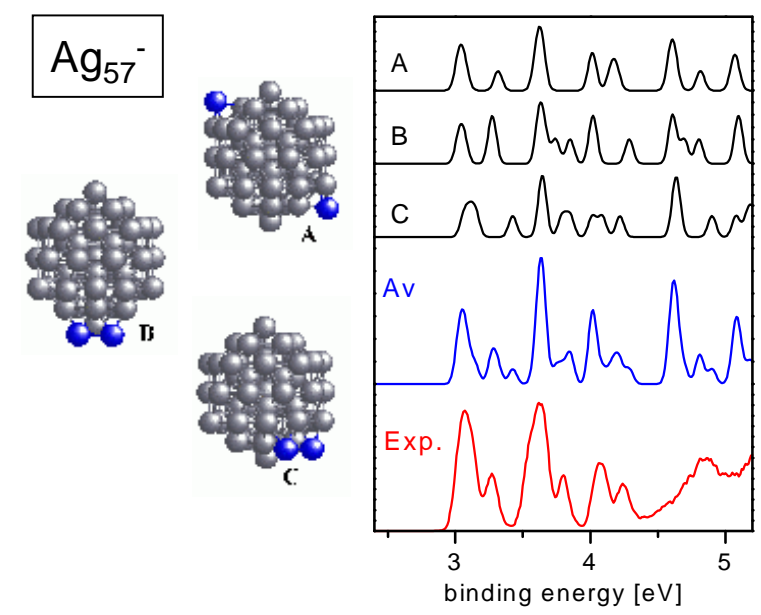

FIG. 3: Three icosahedral-based candidate structures $(\mathrm{A}, \mathrm{B}, \mathrm{C})$ of $\mathrm{Ag}_{57}^{-}$and their calculated DOS as well as an average of $\mathrm{A}, \mathrm{B}$ and $\mathrm{C}(\mathrm{AV})$ and the experimental photoelectron spectrum (Exp.). The calculated energies of the isomers $\mathrm{A}$ and $\mathrm{B}$ with respect to the ground state isomer $\mathrm{C}$ are 0.63 $\mathrm{eV}$ and $0.23 \mathrm{eV}$, respectively.

relativistic element below Fermium" [30], displaying a strong outer shell (6s) contraction and a reduced $5 \mathrm{~d}-6 \mathrm{~s}$ energy gap. This leads to significant s-d hybridization and direct d-d bonding effects, which for instance are also responsible for the anomalous, planar groundstate structures of anionic $\mathrm{Au}_{N}^{-}$clusters up to about $N=12$ [11, 12, 13]. The most obvious consequences of these relativistic effects are a shortening of the interatomic bond lengths and a drastic increase of the bulk modulus. In a calculation of the $\mathrm{Au}_{2}$ potential energy curve we have obtained an increase of the curvature from $5.3 \mathrm{eV} / \AA^{2}$ to $11.6 \mathrm{eV} / \AA^{2}$ when including relativistic effects. The same trend was reported earlier for the calculated bulk modulus of crystalline $\mathrm{Au}$, where a relativistic treatment increased the value from $108 \mathrm{GPa}$ to $182 \mathrm{GPa}[31]$. A recent general study of metal cluster structures demonstrated that it is mainly this high bulk modulus which leads to the different behaviour of gold and silver clusters [29].

The fact that $\mathrm{Au}_{55}^{-}$prefers a low-symmetry structure can therefore be clearly identified as a relativistic effect. This demonstrates the important influence of relativity on the structure of mesoscopic heavy atom particles.

This work was supported by the Academy of Finland and the Deutsche Forschungsgemeinschaft. The experimental work was done by O. Kostko, N. Morgner, M. Astruc Hoffmann 
and B. v. Issendorff. Computations were performed by H.Häkkinen at the CSC-Scientific Computing Ltd. in Espoo, Finland, and by M.Moseler at HLRS in Stuttgart and NIC in Jülich.

[1] W. deHeer, Rev. Mod. Phys. 65, 611 (1993).

[2] T.P.Martin, Phys. Rep. 273, 199 (1996).

[3] M. Haruta, Catalysis Today 36, 153 (1997).

[4] A. Sanchez et al., J. Phys. Chem. A 103, 9574 (1999).

[5] J.F. Hainfield, Science 236, 450 (1987).

[6] W.L. Barnes et al., Nature 424, 824 (2003).

[7] C.Y. Cha et al., J. Chem. Phys. 99, 6308 (1993).

[8] H. Handschuh et al., J. Chem. Phys. 102, 6406 (1995).

[9] J. Tiggesbäumker et al., Chem. Phys. Lett. 190, 42 (1992).

[10] V. Bonacic-Koutecky et al., J. Chem. Phys. 110, 3876 (1999).

[11] H. Häkkinen et al., Phys. Rev. Lett. 89, 033401 (2002).

[12] F. Furche et al., J. Chem. Phys. 117, 6982 (2002).

[13] H. Häkkinen et al., J. Phys. Chem. A 107, 6168 (2003).

[14] X.Li et al., Science 299, 864 (2003).

[15] D. Reinhard et al., Phys. Rev. B 55, 7868 (1997).

[16] L.D. Marks, Rep.Prog.Phys. 57, 603 (1994).

[17] G. Alameddin et al., Chem. Phys. Lett. 192, 122 (1992).

[18] K.J. Taylor et al., J. Chem. Phys. 96, 3319 (1992).

[19] G. Wrigge et al., Phys.Rev. A 65, 063201 (2002).

[20] M. Astruc et al., Phys.Rev.B 66, 041404(R) (2002).

[21] S. Krückeberg et al., Eur. Phys. J D 9, 169 (1999).

[22] A. Herlert et al., Journal of Electron Spectroscopy and Related Phenomena 106, 179 (2000).

[23] R. N. Barnett and U. Landman, Phys. Rev. B 48, 2081 (1993).

[24] J.P. Perdew et al., Phys. Rev. Lett. 77, 3865 (1996).

[25] N. Troullier, and J.L. Martins, Phys. Rev. B 43, 1993 (1991).

[26] The Cambridge Cluster Database, D.J. Wales et al., see URL 
http://brian.ch.cam.ac.uk/CCD.html and references therein.

[27] We call these structures icosahedral although the partial occupation of the uppermost $\mathrm{G}_{g}$ shell leads to a Jahn-Teller (JT) deformation; due to the rigidity of the lattice this deformation is, however, very small. In our unconstrained MD optimization we find JT-induced changes of the bondlengths of less than $2 \%$; this results in a total width e.g. of the $\mathrm{G}_{g}$ derived band of only $0.04 \mathrm{eV}$.

[28] H.P. Cheng et al., Phys. Rev. B 43, 10647 (1991).

[29] J.M. Soler et al., Phys. Rev. B 61, 5771 (2000).

[30] P. Pyykkö, Chem. Rev. 88, 563 (1988).

[31] C. Elsässer et al., J.Phys.: Condens. Mat. 2, 4371 (1990). 